Ann. Biol. anim. Bioch. Biophys., I968, 8 (4), 565-577.

\title{
ISOLEMENT, PURIFICATION ET PROPRIÉTÉS D'UNE PROTEASE EXOCELLULAIRE DE MICROCOCCUS CASEOLYTICUS
}

\author{
M. DESMAZEAUD et J. HERMIER \\ Station centrale de Recherches laitières et de Technologie des Produits animaux, \\ Centre national de Recherches zootechniques, 78 -Jouy-en-Josas \\ Institut national de la Recherche agronomique
}

\section{SOMMAIRE}

L'activité protéolytique exocellulaire de $M$. caseolyticus est attribuée à une protéase unique qui a été purifiée 40 fois par précipitation au sulfate d'ammonium à $85 \mathrm{p}$. 100 de saturation, puis par absorption négative sur D.E.A.E.-cellulose en tampon Tris-maléate $0,05 \mathrm{M}$, suivie d'une chromatographie sur D.E.A.E.-cellulose en tampon Tris-maléate $0,005 \mathrm{M}$ et d'une chromatographie sur Sephadex G 100. La préparation purifiée de protéase est homogène à l'ultracentrifugation.

La protéase a les caractères d'un métallo-enzyme : les ions citrate et l'EDTA l'inhibent à I00 p. Ioo mais les ions $\mathrm{Ca}^{++}$et $\mathrm{Sr}^{++}$assurent une réactivation partielle (60 p. Ioo pour les ions $\left.\mathrm{Ca}^{++}\right)$. Les composés réducteurs l'inhibent partiellement ; le DFP et l'acide iodoacétique n'exercent aucune inhibition. Le poids moléculaire déterminé par filtration sur Séphadex G 100 est de $38000 \pm 3000$. Le coefficient d'extinction $\mathrm{E}$ à $20^{\circ} \mathrm{C}$ en tampon Tris-maléate $0,005 \mathrm{M}$ à pH 7 est $\mathrm{E}_{1 \mathrm{~cm}}^{1 \%}=8,95$ à $280 \mathrm{~m} \mu$.

Cette protéase présente une grande affinité vis-à-vis de la caséine $\left(\mathrm{K}_{m}=0, \mathbf{1 2 8} \mathrm{p}\right.$. I00) ; l'hémoglobine dénaturée $\left(\mathrm{K}_{m}=2,2 \mathrm{p}\right.$. I00) et la $\beta$-lactoglobuline $\left(\mathrm{K}_{m}=9 \mathrm{p}\right.$. I00) sont aussi des substrats. La température optimum est de $5^{\circ} \mathrm{C}$ (chaleur d'activation $\Delta \mathrm{H}=8640$ calories par mole à $50^{\circ} \mathrm{C}$ ). Le $\mathrm{pH}$ optimum est de 7,40.

Même en présence des ions $\mathrm{Ca}^{++}$cette protéase neutre est rapidement inactivée par la chaleur (chaleur d'activation $\Delta H \delta=27,360$ kilocalories par mole à $5^{\circ} \mathrm{C}$ ). En l'absence d'ions $\mathrm{Ca}^{++}$l'activité n'est conservée que dans une zone de $\mathrm{pH}$ très étroite (optimum 7,00 ) ; en présence d'ions $\mathrm{Ca}^{++}$ l'enzyme est stable en milieu alcalin jusqu'à $\mathrm{pH} 8,50$. L'instabilité de la protéase est due à une autolyse de la protéine qui est inhibée par les ions $\mathrm{Ca}^{++}$.

\section{INTRODUCTION}

Nous avons montré dans la première partie de ce travail (DESMAZEAUD et HERMIER, I968) qu'une souche de $M$. caseolyticus stimulatrice de la croissance de certaines

Abréviations : Tris : tris(hydroxyméthyle) aminométhane ;

EDTA : éthylène diamine tétracétique.

D.E.A.E. : diéthylaminoéthyle. 
bactéries lactiques, produit dans certaines conditions de culture un système protéolytique exocellulaire.

Les protéases exocellulaires n'ont été complètement purifiées que chez un nombre limité de genres bactériens (HAGrHARA et al., I958; MoRIHARA, I963 ; Mizusawa, I964; McConn et al., I964). En ce qui concerne le genre Micrococcus, quelques caractéristiques de l'activité protéolytique produite dans le milieu de culture ont été déterminées chez $M$. freudenreichii (HuSAIN et MCDONALD, I958) et $M$. caseolyticus (POZNANSKI, LENOIR et MOcQUOT, I965).

Dans le présent travail nous avons complètement purifié par chromatographie sur colonne, le système protéolytique produit par $M$. caseolyticus. Nous en avons, d'autre part, caractérisé certaines propriétés et montré en particulier que son instabilité a pour origine une forte activité autoprotéolytique qui est inhibée par les ions calcium.

\section{I. - MATÉRIEL ET MÉTHODES}

\section{Souche bactérienne}

Les caractères de la souche $\mathrm{n}^{0} 9^{6}$ de $M$. caseolyticus utilisée dans ce travail sont décrits dans la première partie (DESMAZEAUD et HERMIER, i 968 ).

\section{Production du système protéolytique}

Les conditions de culture sont celles décrites précédemment (DESMAZEAUd et HERMIER, 1968) pour la croissance dans le milieu TGCa maintenu à $\mathrm{pH}$ constant. Après 80 heures de culture à $30^{\circ} \mathrm{C}$, les cellules sont séparées du milieu par centrifugation à $4^{\circ} \mathrm{C}$ pendant $3^{\circ}$ minutes à $8000 \mathrm{~g}$ dans une centrifugeuse réfrigérée Sorvall.

\section{Purification du système protéolytique}

Des essais préliminaires ayant montré que les ions calcium sont nécessaires à la stabilité de l'enzyme en solution, le tampon Tris-maléate (au pH utilisé pour mettre la protéase en solution au cours de la purification) contient toujours du chlorure de calcium à la concentration de $1,5 \cdot 10^{-3} \mathrm{M}$. Toutes les opérations de purification sont effectuées à la température ambiante à l'exception des dialyses et des centrifugations qui sont faites à $4^{\circ} \mathrm{C}$.

Les précipitations par le sulfate d'ammonium sont réalisées par dialyse : la solution enzymatique est dialysée pendant 24 heures à $4^{\circ} \mathrm{C}$ contre une solution sursaturée de sulfate d'ammonium dont la quantité est calculée de façon à obtenir une concentration égale à $85 \mathrm{p}$. roo de la saturation quand l'équilibre des concentrations est réalisé. Au cours de la dialyse quand tout le sulfate d'ammonium est passé en solution, le $\mathrm{pH}$ est ajusté à 7,0 par addition d'ammoniaque $6 \mathrm{~N}$.

Cette méthode de précipitation a été préférée à la méthode d'addition directe du sulfate d'ammonium dans la solution à précipiter, car elle permet l'o'stention d'un précipité plus gros et par suite plus facilement recueilli par centrifugation à 30 ooo $g$ pendant une heure. Le précipité est ensuite dissous dans le tampon Tris-maléate et dialysé pendant 24 heures contre ce même tampon.

\section{Méthodes et techniques de mesure}

L'activité protéolytique est mesurée conformément à la technique décrite dans la première partie de ce travail (DESMAZEAUd et HERMIER, I968). L'activité spécifique est définie comme le nombre d'unités d'activité protéolytique par $\mathrm{mg}$ de protéines ; celles-ci sont dosées par la méthode spectrophotométrique de WARBURG et Christian (I94I). 
La coloration à la ninhydrine est effectuée d'après la méthode de Moore et STEIN (1948). La courbe standard des densités optiques en fonction de la concentration est obtenue avec de la leucine pure en tampon citrate $0, \mathrm{I} \mathrm{M}$ à $\mathrm{pH} 5$.

Le test d'homogénéité est réalisé dans une ultracentrifugeuse Beckman, modèle $\mathrm{E}$, opérant à 59780 tours par minute à $20^{\circ} \mathrm{C}$. La solution enzymatique (en tampon Tris-maléate de sodium $0,005 \mathrm{M}$, à $\mathrm{pH} 7,0$ ) est à une concentration de I p. Ioo; le volume de la cellule étant de $0,8 \mathrm{ml}$.

Le poids moléculaire est déterminé par filtration sur gel (ANDREWS, I964; WHITAKER, 1963). La colonne de Sephadex G $100(25 \mathrm{~cm} \times 2 \mathrm{~cm})$ est préparée en tampon Tris-maléate de sodium o,005 M à $\mathrm{pH} 7,0.4 \mathrm{ml}$ d'une solution de protéine à 0,1 p.I00 dans le même tampon sont déposés au sommet de la colonne puis élués. Le volume d'élution est déterminé à partir de la courbe d'enregistrement de l'absorption de l'éluat à $280 \mathrm{~m} \mu$.

\section{II. - RÉSULTATS}

\section{A. - Isolement et purification de la protéase}

\section{Ire étape : Précipitation par le sulfate d'ammonium à 85 p. 100 de saturation.}

Les I $500 \mathrm{ml}$ du surnageant de culture après élimination des cellules bactériennes sont précipités par le sulfate d'ammonium selon la méthode décrite dans le chapitre "Matériel et Méthodes ". Le précipité est redissous dans $40 \mathrm{ml}$ de tampon Tris-maléate $0,05 \mathrm{M}$ à $\mathrm{pH} 7,0$, puis dialysé pendant 40 heures contre ce même tampon.

\section{2 e étape : Première chromatographie sur colonne de D.E.A.E.-cellulose.}

Cette étape de la purification, ainsi que la suivante, exploitent le fait que le système protéolytique étudié n'est pas retenu sur une colonne de D.E.A.E.-cellulose équilibrée avec du tampon Tris-maléate $0,05 \mathrm{M}$ alors qu'il est retenu quand la colonne est équilibrée avec le même tampon $0,005 \mathrm{M}$.

Les $40 \mathrm{ml}$ de la solution enzymatique contenant $25 \mathrm{mg}$ par $\mathrm{ml}$ de protéines sont déposés au sommet de la colonne de D.E.A.E.-cellulose $(30 \mathrm{~cm} \times 2 \mathrm{~cm})$ équilibrée avec du tampon Tris-maléate $0,05 \mathrm{M}$. La colonne est éluée par passage du même tampon et les fractions de l'éluat montrant une activité protéolytique sont rassemblées et précipitées par le sulfate d'ammonium. Le précipité obtenu est ensuite redissous dans $\mathrm{I} 7 \mathrm{ml}$ de tampon Tris-maléate $0,005 \mathrm{M}$ de $\mathrm{pH} 7,0$ et dialysé 24 heures contre ce même tampon.

\section{3e étape : Deuxième chromatographie sur colonne de D.E.A.E.-cellulose.}

Les $17 \mathrm{ml}$ de solution enzymatique contenant $140 \mathrm{mg}$ de protéines sont déposés sur une colonne de D.E.A.E.-cellulose $(26 \mathrm{~cm} \times \mathrm{I} \mathrm{cm})$ équilibrée avec du tampon Tris-maléate $0,005 \mathrm{M}$ à $\mathrm{pH} 8,0$. La colonne est éluée par passages successifs de solutions de chlorure de sodium à concentration croissante en tampon Tris-maléate $0,005 \mathrm{M}$ à pH 7,0. Comme on peut le voir sur la figure $\mathrm{I}$, la presque totalité du système protéolytique est éluée par la solution de $\mathrm{NaCl}$ à $\mathrm{o}, \mathrm{I} \mathrm{M}$. La fraction éluée par la solution de $\mathrm{NaCl}$ o,2 $\mathrm{M}$ montre une faible activité protéolytique.

Les fractions correspondant à la partie symétrique du pic d'activité protéolytique sont rassemblées et précipitées par le sulfate d'ammonium à 85 p. Ioo de satu- 
ration. Le précipité recueilli est redissous dans $6 \mathrm{ml}$ de tampon Tris-maléate $0,005 \mathrm{M}$ à $\mathrm{pH} 7,0$ et dialysé 24 heures contre ce même tampon.

4e étape : Filtration sur colonne de Sephadex G 100.

Six $\mathrm{ml}$ de la solution enzymatique contenant $23 \mathrm{mg}$ de protéines sont déposés au sommet d'une colonne $(25 \mathrm{~cm} \times 2 \mathrm{~cm})$ de Sephadex $\mathrm{G}$ Ioo préparée en tampon

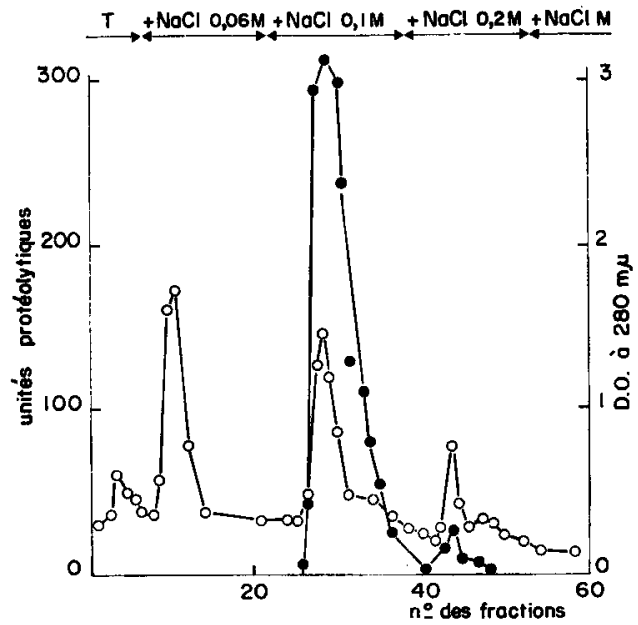

FIG. I. - Chromatographie sur DEAE-cellulose équilibrée en tampon Tris-maléate $0,005 M$ dI $p H$ $\mathrm{T}=$ Tampon de départ : Tris-maléate $0,005 \mathrm{M}+\mathrm{CaCl}_{\mathbf{2}} \mathrm{I}, 5 \cdot \mathrm{IO}^{-\mathbf{3}} \mathrm{M}$ 0 : courbe d'élution des protéines (densité optique à $280 \mathrm{m \mu}$ ) - : activité protéolytique (unités par ml)

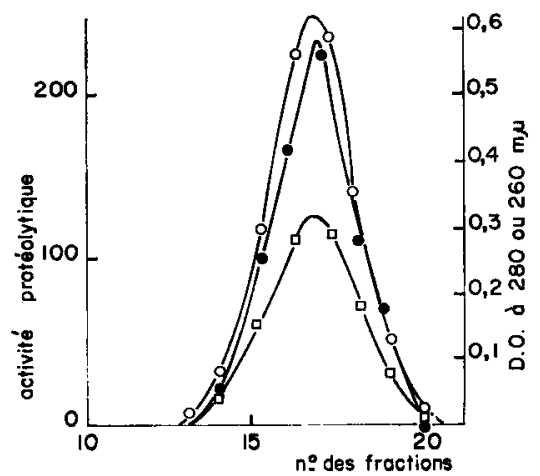

FIG. 2. - Chromatographie sur Sephadex G 100 de la protéase purifiée Éluant : tampon Tris-maléate $0,005 \mathrm{M}+\mathrm{CaCl}_{2} \mathrm{I}, 5 \cdot 10^{-\mathrm{a}} \mathrm{M}$ - - $\square-\quad$ : densité optique à $260 \mathrm{~m} \mu$

—-_- :

Tris-maléate $0,005 \mathrm{M}$. Toutes les protéines sont éluées par ce même tampon en un pic unique qui est symétrique. Le pic d'activité protéolytique coinncide rigoureusement 
avec le pic des protéines comme il est montré dans la figure 2. La préparation est donc homogène vis-à-vis de la filtration sur gel.

Les rendements des différentes étapes de la purification sont présentés dans le tableau $\mathrm{I}$.

TABLEAU I

Processus d'isolement et bilan de la purification

de la protéase exocellulaire de M. caseolyticus

\begin{tabular}{|c|c|c|c|c|c|c|c|}
\hline & $\begin{array}{l}\text { Volume } \\
(\mathrm{ml})\end{array}$ & $\begin{array}{c}\text { Activité } \\
\text { protéolytique } \\
\text { unités/ml }\end{array}$ & $\begin{array}{l}\text { Unités } \\
\text { totales }\end{array}$ & $\begin{array}{l}\text { Protéines } \\
\text { en } \\
\text { grammes }\end{array}$ & $\begin{array}{l}\text { Activité } \\
\text { spécifique } \\
\text { unités/mg }\end{array}$ & $\begin{array}{c}\text { Rendement } \\
(\%)\end{array}$ & $\begin{array}{l}\text { Purifi- } \\
\text { cation }\end{array}$ \\
\hline Surnageant de culture & 1590 & 305 & 485000 & 7,79 & 62,2 & 100 & 1 \\
\hline $\begin{array}{c}\text { Précipitation } \\
\text { par } \mathrm{SO}_{4}\left(\mathrm{NH}_{4}\right)_{2} \\
85 \% \text { saturation }\end{array}$ & 41 & 7810 & 320000 & 1,02 & 312 & 66 & 5 \\
\hline $\begin{array}{l}\text { Absorption négative } \\
\text { sur DEAE-cellulose }\end{array}$ & 320 & 699 & 223000 & 0,138 & 1620 & 49 & 26 \\
\hline $\begin{array}{c}\text { Précipitation } \\
\text { par } \mathrm{SO}_{4}\left(\mathrm{NH}_{4}\right)_{2} \\
85 \%{ }^{-} \text {saturation }\end{array}$ & 11,2 & 13610 & 152900 & 0,091 & 1680 & 31,5 & 27 \\
\hline $\begin{array}{l}\text { Chromatographie } \\
\text { sur DEAE-cellulose }\end{array}$ & 30 & 2425 & 72800 & 0,033 &, 2960 & 15 & 38 \\
\hline $\begin{array}{c}\text { Précipitation } \\
\text { par } \mathrm{SO}_{4}\left(\mathrm{NH}_{4}\right)_{2} \\
85 \% \text { saturation }\end{array}$ & 6,10 & 9090 & 55200 & 0,023 & 2390 & 11,4 & 38,4 \\
\hline $\begin{array}{l}\text { Chromatographie } \\
\text { sur Sephadex G } 100\end{array}$ & 35 & $9: 0$ & 33990 & 0,013 & 2490 & 7 & 40 \\
\hline
\end{tabular}

\section{B. - Propriétés physico-chimiques de la protéase}

La préparation purifiée de la protéase de $M$. caseolyticus est homogène à l'ultracentrifugation car l'image obtenue ne présente qu'un pic symétrique.

Le spectre d'absorption en ultraviolet de la protéase en tampon Tris-maléate $0,005 \mathrm{M}$ à $\mathrm{pH} 7,0$ et à $20^{\circ} \mathrm{C}$, présente un maximum d'absorption à $276 \mathrm{~m} \mu$ et un minimum d'absorption à $25^{8} \mathrm{~m} \mu$ (fig. 3). Le coefficient d'extinction $\mathrm{E}$ à $20^{\circ} \mathrm{C}$ est $\mathrm{E}_{\mathrm{I}}^{\mathrm{r}} \mathrm{cm}=8,95$ à $280 \mathrm{~m} \mu$.

Le poids moléculaire de la protéase neutre est estimé à $38000 \pm 3000$ par comparaison de son volume d'élution d'une colonne de Sephadex $\mathrm{G}$ roo, avec celux de la trypsine (poids moléculaire : 24000 ), pepsine (poids moléculaire : 36000 ) et sérumalbumine (poids moléculaire 69000 ) (fig. 4). La trypsine, la pepsine et la sérumalbumine sont des produits Nutritional Biochemicals Corporation. 


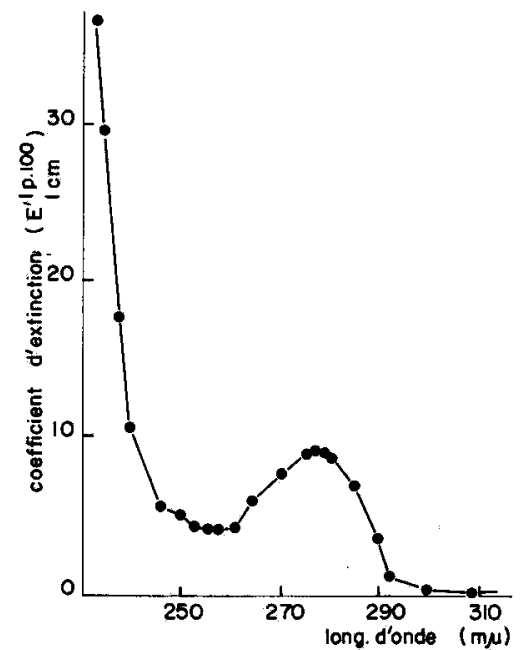

FIG. 3. - Spectre d'absorption ultraviolet de la protéase.

Tampon : Tris-maléate $0,005 \mathrm{M}+\mathrm{CaCl}_{\mathbf{2}}$ $1,5^{\cdot 10^{-3}} \mathrm{M}$ pH 7,0 à $20^{\circ} \mathrm{C}$

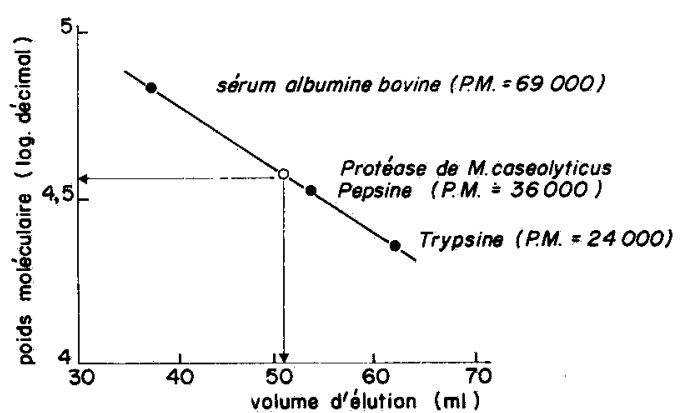

FIG. 4. - Estimation du poids moléculaire de la protéase neutre exocellulaire de M. caseolyticus

\section{C. - Stabilité de la protéase}

Influence $d u p H$ et des ions calcium.

La solution enzymatique sans calcium est obtenue en dialysant contre du tampon Tris-maléate o,005 M le volume de solution désiré. En absence d'ions calcium, la protéase n'est stable qu'au voisinage de $\mathrm{pH} 7$. Après $30 \mathrm{mn}$ à $30^{\circ} \mathrm{C}$ 1'enzyme est déjà partiellement inactivé à $\mathrm{pH} 8,0$ ou à $\mathrm{pH} 6, \mathrm{o}$. Par contre, en présence d'ions calcium $\mathrm{I}, 5 \cdot \mathrm{IO}^{-3} \mathrm{M}, 1^{\prime}$ 'enzyme est stable dans les mêmes conditions entre $\mathrm{pH} 7,0$ et 8,5 mais reste instable quand le $\mathrm{pH}$ est inférieur à 7,0 (fig. 5).

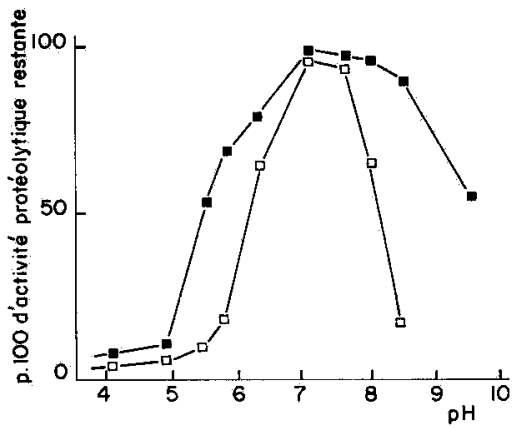

FIG. 5. - Influence $d u$ pH et des ions calcium sur la stabilite de la protéase neutre de M. caseolyticus

$\mathrm{pH}$ io : Tampon glycine- $\mathrm{NaOH}$ o, $05 \mathrm{M}$

$\mathrm{pH}$ : Tampon Tris-HCl o,05 M

$\mathrm{pH} 5$ à 8,60: Tampon Tris-maléate o,o5 M

$\mathrm{pH} 4$ : Tampon acétate $0,05 \mathrm{M}$

L'enzyme est incubé 30 minutes à $30^{\circ} \mathrm{C}$ aux différents $\mathrm{pH}$.

Les ions calcium sont éliminés de la solution de protéase par dialyse à $\mathrm{pH} 7$ à $4^{\circ} \mathrm{C}$.

- : enzyme avec calcium $\left(\mathrm{CaCl}_{2} \mathrm{I}, 5.10^{-8} \mathrm{M}\right)$ 
L'influence de la concentration des ions calcium sur la stabilité de l'enzyme incubé à $30^{\circ} \mathrm{C}$ en tampon Tris-maléate à $\mathrm{pH} 7,0$ pendant 20 heures et 44 heures est montrée dans la figure 6 . La perte d'activité devient notable quand la concentration en ions calcium est inférieure à $\mathrm{I}, 5 \cdot 1 \mathrm{O}^{-3} \mathrm{M}$.

Cette instabilité de la protéase peut être attribuée à une forte activité autoprotéolytique de 1'enzyme, comme cela a été montré chez d'autres protéases (WU et LASKOWSKI, I956; MCCoNn et al., I964). Enn effet, à la fin de la période d'incubation de 48 heures, on constate une augmentation dans la solution enzymatique du matériel colorable par la ninhydrine, cette augmentation étant d'autant plus forte que l'inactivation de l'enzyme est plus importante, donc que la concentration des ions calcium est plus faible (fig. 6). Cette corrélation entre la perte d'activité et l'apparition de matériel colorable à la ninhydrine peut être montrée au cours de 1'incubation à $30^{\circ} \mathrm{C}$ dans des conditions où l'enzyme perd rapidement son activité $(\mathrm{pH} 8,4$, concentration en ions calcium $5 \cdot 10^{-5} \mathrm{M}$ ).

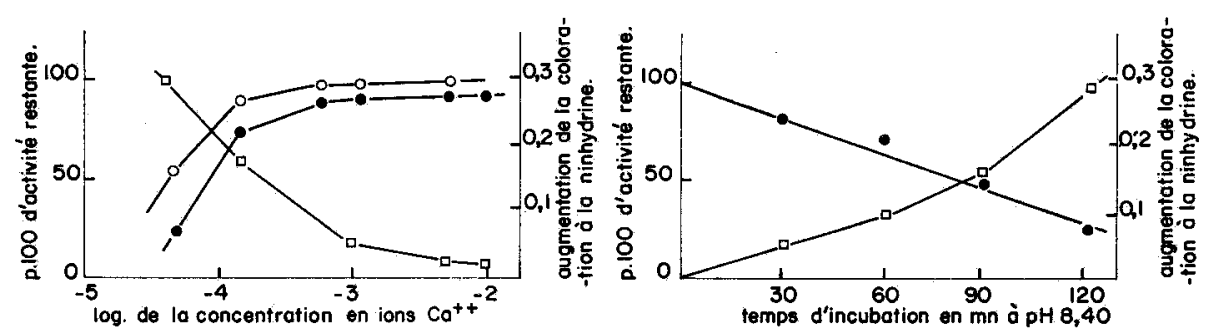

FIG. 6. -Étude de lautolyse de la protéase neutre

Partie $A-\exists-\square-$ : augmentation de la coloration après colorimétrie à la ninhydrine après 44 heures d'incubation

-O- - : \% d'activité protéolytique restante après incubation à $30^{\circ} \mathrm{C}$ à $\mathrm{pH} 7$ pendant 20 heures;

- - - : : \% d'activité protéolytique restante après 44 heures.

Concentration en protéase : $390 \mu \mathrm{g}$ dans $1,5 \mathrm{ml}$ de tampo., Tris-maléate

Partie $B \quad \square-\square \quad$ : augmentation de la coloration après colorimśtrie à la ninhydrine :

- : \% d'activité protéolytique restante.

L'influence stabilisante des ions calcium est aussi observée dans le cas de l'inactivation de l'enzyme par chauffage puisqu'elle est plus rapide en tampon phosphate $0,067 \mathrm{M}$ qu'en tampon Tris-maléate $0,005 \mathrm{M}$ contenant des ions calcium $\mathrm{I}, 5 \cdot 10^{-3} \mathrm{M}$.

L'énergie apparente d'activation $\mathrm{E}$ et la chaleur d'activation $\Delta \mathrm{H}$ pour la réaction d'inactivation de la protéase par la chaleur ont été calculées d'après la méthode proposée par Akiba et Fukimbara (1967). Les valeurs obtenues sont les suivantes :

$\mathrm{E}=28$ ooo calories par mole et $\Delta \mathrm{H}=27,36 \mathrm{Kcal}$ par mole à $50^{\circ} \mathrm{C}$.

\section{D. - Facteurs commandant la vitesse de protéolyse de la caséine}

\section{Influence $d u p H$.}

La protéase de $M$. caseolyticus rentre dans la catégorie des protéases neutres puisque la vitesse de protéolyse est maximale à $\mathrm{pH} 7,40$ (fig. 7 ). Cette valeur est sensiblement la même que celle du $\mathrm{pH}$ de stabilité maximale de la protéase. La vitesse de protéolyse décroît rapidement quand le $\mathrm{pH}$ est inférieur à 6,50 ou supérieur à 8,50 . 


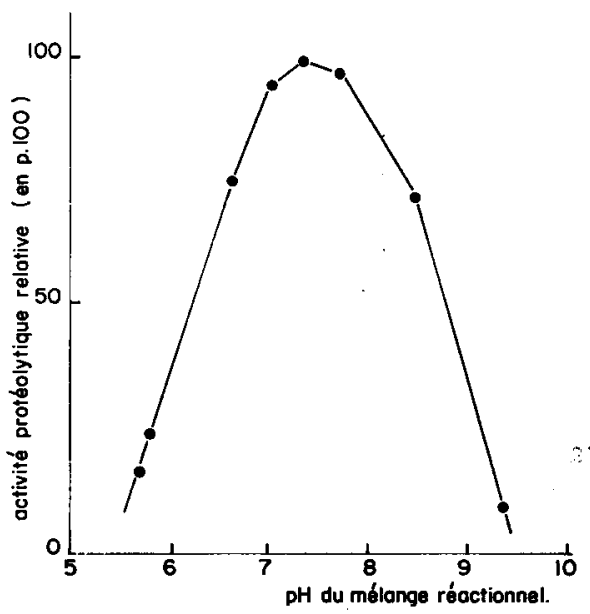

Fig. 7. - Infiuence du $p H$ réactionnel sur la vitesse de la protélyse de la caséine Température d'incubation : $30^{\circ} \mathrm{C}$

Tampon Tris-maléate o,o5 $\mathrm{M}: \mathrm{pH} 5,0$ à $\mathrm{pH} 8,60$

Tampon Tris- $\mathrm{HCl} 0,05 \mathrm{M}: \mathrm{pH} 9,40$

\section{Influence de la température.}

La figure 8 montre la variation de la vitesse de la protéolyse en fonction de la température. Dans les conditions employées (durée de réaction : Io $\mathrm{mn}$ ), la température optimale apparente est de $50^{\circ} \mathrm{C}$. En fait, elle est probablement un peu supérieure puisque dans les conditions employées $25 \mathrm{p}$. roo de l'activité sont déjà perdus à cette température (voir fig. 9). La vitesse de la réaction en fonction de la température suit la loi,d'ARRHENIUS (fig. 8 a). L'énergie apparente d'activation E est de 9 IOo calories par mole et la chaleur d'activation de 8460 calories par mole à $50^{\circ} \mathrm{C}$.
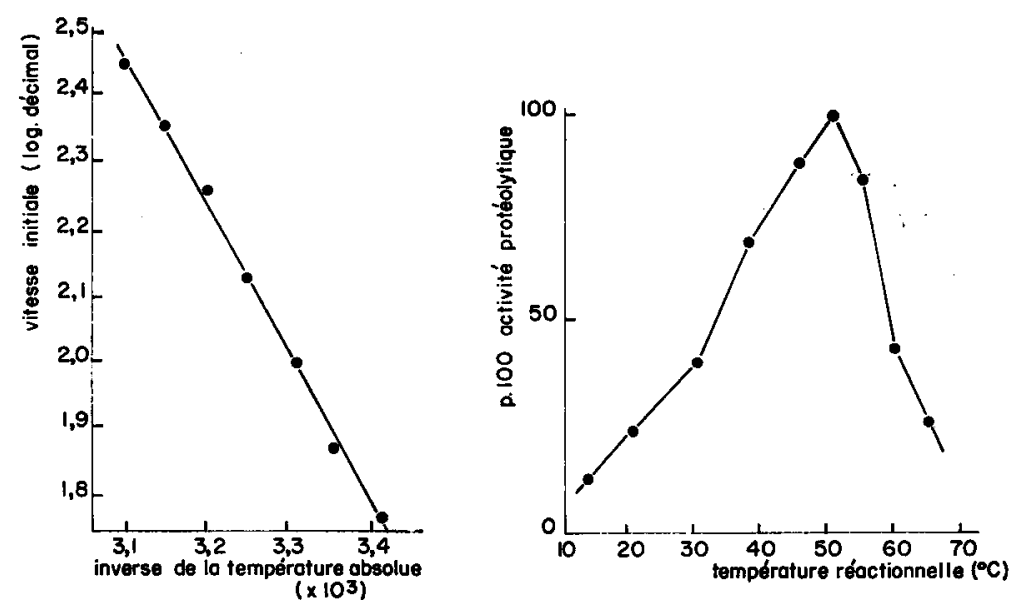

FIG. 8. - Influence de la température sur la vitesse de la protéolyse de la caséine par la protéase neulre

a) Représentation graphique de la loi d'Arrhenius dans le cas de l'hydrolyse de la caséine par la protéase neutre 


\section{Influence de la concentration en substrat.}

La vitesse d'hydrolyse en fonction de la concentration en substrat suit la loi de Henri (IgO3) et Michaelis-Menten (I9I3). La constante de Michaelis $\left(\mathbf{K}_{m}\right)$ a été déterminée graphiquement suivant le système de coordonnées de LINEWEAVERBurk (1934) (fig. ro). Elle a pour valeur 0,128 p. roo à $\mathrm{pH}$ 7,0 dans le cas de la caséine isoélectrique.

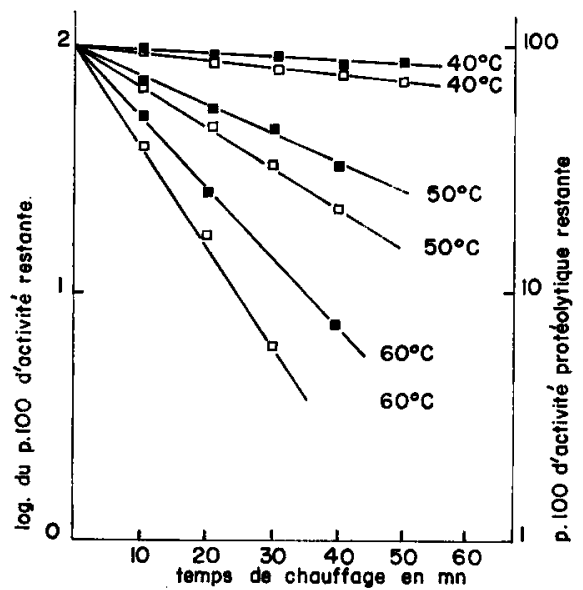

FIG. 9. - Etude de la stabilité thermique de la protéase

-n- : Chauffage en tampon Tris-maléate $0,05 \mathrm{M}+\mathrm{CaCl}_{2} \mathrm{1}, 5 \cdot 10^{-8} \mathrm{M}$ à $\mathrm{pH} 7,0$

- $-\square-$ : Chauffage en tampon phosphate $0,066 \mathrm{M}$ aे $\mathrm{pH}_{7,0}$

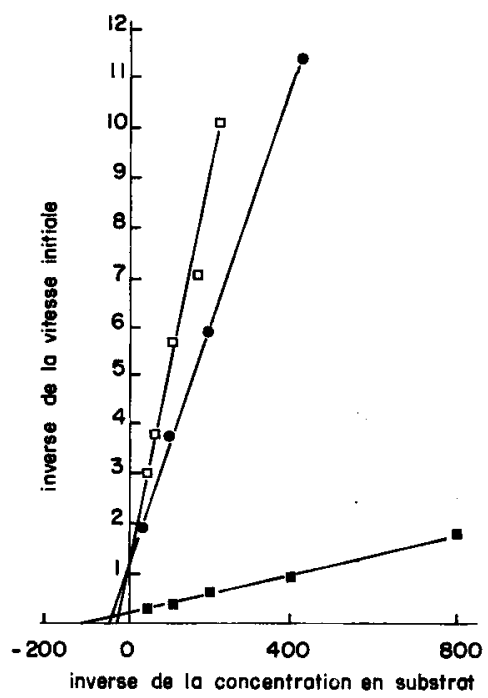

FIG. Io. - Delermination graphique de la constante de MichaELIs dans le cas de l'hydrolyse de trois proteines substrats

- : caséine isoélectrique -_- : íémoglobine dénaturée ¿- : $\quad$-lactoglobuline

L'hémoglobine dénaturée et la $\beta$-lactoglobuline (Produits Nutritional Biochemicals Corporation) sont aussi des substrats de la protéase de $M$. caseolyticus; les constantes de MICHAEIIs correspondantes ont pour valeur 2,2 p. IOO et 9 p. IOO à $\mathrm{pH} 7,0$.

Dans le cas de la sérum-albumine de bœuf, la réaction est trop faible pour pouvoir être mesurée avec suffisamment de précision.

\section{E. - Inhibiteurs de l'activité protéolytique}

L'activité protéolytique a été mesurée après $60 \mathrm{mn}$ d'incubation à $30^{\circ} \mathrm{C}$ dans une solution à $\mathrm{pH} 7,0$ de tampon Tris-maléate $0,005 \mathrm{M}$ du composé essayé.

L'activité protéolytique est complètement inhibée par les ions $\mathrm{Fe}^{++}, \mathrm{Hg}^{++}$, $\mathrm{Cu}^{++}, \mathrm{Ag}^{++}$, fortement inhibée par les cations $\mathrm{Cd}^{++}, \mathrm{Zn}^{++}$et $\mathrm{Pb}^{++}$à la concentration $2 \cdot \mathrm{IO}^{-3} \mathrm{M}$ et les ions $\mathrm{Co}^{++}$et $\mathrm{Mn}++$ à la concentration $2 \cdot \mathrm{IO}^{-2} \mathrm{M}$ (tabl. 2). Les ions $\mathrm{Ca}^{++}$, $\mathrm{Sr}^{++}, \mathrm{Mg}^{++}, \mathrm{Na}^{++}$et $\mathrm{K}^{+}$ne provoquent pas d'inhibition même à une concentration 2. $\mathrm{IO}^{-\mathbf{2}} \mathrm{M}$. 
La protéase est inhibée par des agents chélateurs (tels que l'éthylènediaminetétracétate, le 8-quinolinol et les ions citrates) mais pas par les ions oxalates (tabl. 3).

\section{TABLEAU 2}

Action inhibitrice des cations sur l'activité protélytique de la protéase neutre de Micrococcus caseolyticus

\begin{tabular}{|c|c|c|}
\hline & \multicolumn{2}{|c|}{$\%$ de l'activité initiale } \\
\hline & $2 \times 10^{-3} \mathrm{M}$ & $2.10^{-2} \mathrm{M}$ \\
\hline $\mathrm{Fe}^{++} \ldots \ldots \ldots \ldots \ldots$ & 0 & \\
\hline $\mathrm{Hg}++\ldots \ldots \ldots \ldots \ldots$ & 0 & \\
\hline $\mathrm{Cu}++\ldots \ldots \ldots \ldots \ldots$ & 0 & \\
\hline $\mathrm{Ag}^{++} \ldots \ldots \ldots \ldots \ldots$ & 0 & \\
\hline $\mathrm{C} \bar{d}++\ldots \ldots \ldots \ldots \ldots$ & 4 & \\
\hline $\mathrm{Zn}^{++} \ldots \ldots \ldots \ldots \ldots$ & 12 & \\
\hline $\mathrm{Pb}^{t+} \ldots \ldots \ldots \ldots \ldots$ & 13 & \\
\hline $\mathbf{M n}^{++} \ldots \ldots \ldots \ldots$ & 75 & 50 \\
\hline $\mathrm{Co}^{++} \ldots \ldots \ldots \ldots$ & 95 & 10 \\
\hline $\mathrm{Sr}^{++} \ldots \ldots \ldots \ldots \ldots$ & 100 & 100 \\
\hline $\mathrm{Mg}^{++} \ldots \ldots \ldots \ldots \ldots$ & 100 & 100 \\
\hline $\mathrm{Ca}^{++} \ldots \ldots \ldots \ldots \ldots$ & 100 & 100 \\
\hline $\mathrm{Na}+\ldots \ldots \ldots \ldots$ & 100 & 100 \\
\hline $\mathbf{K}+\ldots \ldots \ldots \ldots \ldots$ & 100 & 100 \\
\hline
\end{tabular}

TABLEAU 3

Action des inhibiteurs enzymatiques sur l'activité protéolytique de la protéase neutre de Micrococcus caseolyticus

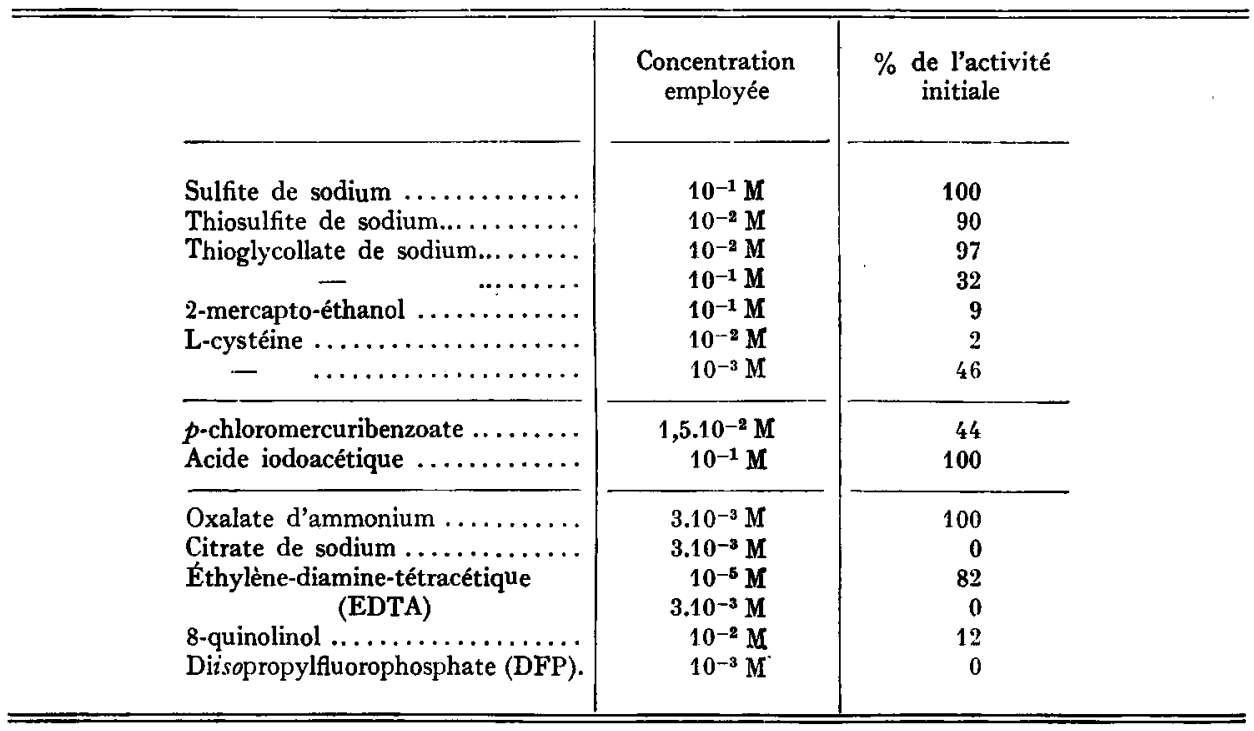


Après traitement par une solution d'EDTA $\mathrm{IO}^{-4} \mathrm{M}$, la protéase est partiellement réactivée par incubation à $30^{\circ} \mathrm{C}$ pendant $60 \mathrm{mn}$ dans une solution de chlorure de calcium ou de chlorure de strontium $2 \cdot \mathrm{IO}^{-2} \mathrm{M}$. On retrouve ainsi respectivement $60 \mathrm{p}$. Ioo et $4^{\circ} \mathrm{p}$. Ioo de l'activité initiale après traitement par les cations $\mathrm{Ca}^{++}$et $\mathrm{Sr}^{++}$.

Le diisopropylfluorophosphate, composé qui réagit avec la sérine, n'inhibe pas l'activité de la protéase (tabl. 3).

Parmi les réactifs du groupe sulfhydryle l'acide iodoacétique ne montre aucune action sur l'enzyme alors que celui-ci est partiellement inactivé par le $p$-chloromercuribenzoate à $I, 5 \cdot 10^{-2} \mathrm{M}$ (tabl. 3).

Enfin, parmi les composés réducteurs le sulfite de sodium ou le thiosulfate de sodium n'affectent pas l'activité enzymatique, alors que le 2-mercaptoéthanol, le thioglycollate de sodium et la L-cystéine à une concentration de ${ }^{-1} 0^{-1} \mathrm{M}$ inhibent l'activité protéolytique. L'effet inhibiteur de la cystéine est encore observable quand celle-ci est à la concentration de $\mathrm{IO}^{-3} \mathrm{M}$.

\section{DISCUSSION}

Le système protéolytique exocellulaire de $M$. caseolyticus est décomposable en deux constituants par chromatographie sur D.E.A.E.-cellulose. Le constituant élué en premier représente la presque totalité de l'activité protéolytique; il se comporte comme une protéine homogène à l'ultracentrifugation et à la filtration sur gel. Le constituant élué en second n'a pas été étudié ; il est très possible qu'il représente non pas une autre protéase, mais un ou plusieurs produits de dégradation du premier constituant formé par auto-protéolyse au cours des premières étapes de la préparation de l'enzyme.

Le processus de purification employé ne permet qu'un faible rendement ( $7 \mathrm{p}$. Ioo, purification : 40 fois) qui a en partie pour origine une perte non reversible de l'activité enzymatique au cours des précipitations par le sulfate d'ammonium; une autolyse partielle au cours des opérations de purification peut aussi contribuer à diminuer le rendement.

Ia protéase de $M$. caseolyticus est remarquable par son instabilité en absence d'ions calcium. Elle nécessite les ions calcium pour sa stabilité à une concentration minimum de $\mathrm{I}, 5 \times 10^{-3} \mathrm{M}$. Cette action protectrice des ions calcium est fréquente chez les protéases bactériennes; elle a été observée en particulier chez les genres proches des microcoques (VALIIER, I966 ; CoLOBERT, I957 ; GoRINI, I950). Comme pour la protéase neutre de $B$. subtilis (MCCoNN, I954) l'action protectrice des ions calcium chez $M$. caseolyticus est due à l'inhibition par ces ions de l'auto-protéolyse de l'enzyme. Il est remarquable que cette inhibition soit obtenue avec des concentrations de chlorure de calcium qui n'ont aucun effet sur l'activité de l'enzyme vis-à-vis d'autres substrats tels que la caséine. Cet effet différentiel des ions calcium pourrait suggérer que le ou les sites actifs de l'enzyme intervenant dans la formation du complexe enzyme-substrat ne sont pas les mêmes quand le substrat est la caséine ou la protéine enzymatique.

La protéase de Micrococcus caseolyticus présente la même spécificité de substrat que celle du système protéolytique non purifié de $M$. freudenreichii (HusaIN et

Annales də Biologie animale. - 1968 . 
MCDonal, $\left.195^{8}\right)$, la même température optimale $\left(5^{\circ} \mathrm{C}\right)$, mais un $\mathrm{pH}$ optimal différent (7,4 chez $M$. caseolyticus ; 6,4 chez $M$. freudenreichii).

L'action des cations et des inhibiteurs de l'activité enzymatique révèle que la protéase de Micrococcus présente les caractères d'un métalloenzyme: inactivation par les agents chélateurs et réactivation par certains ions divalents. Ces caractères rapprochent cet enzyme du système protéolytique de $M$. lysodeikticus (GoRINI, I950) et de la protéinase de Streptococcus faecalis var. liquefaciens (BLEIwEIs et ZIMMERMAN, 1964) mais ce dernier enzyme est réactivé par les ions $\mathrm{Zn}^{++}$et non par les ions $\mathrm{Ca}^{++}$après traitement par les agents chélateurs. Les protéases de $M$. caseolyticus et de Str. faecalis possèdent d'autres propriétés en commun. En dehors des agents chélateurs leur activité est inhibée par les composés réducteurs alors qu'elle ne l'est pas par des agents se combinant avec les groupes sulfhydryles ou la fonction hydroxyle de la sérine (JANSEN et BALIS, I952). Les valeurs de l'énergie d'activation sont comparables ainsi que celles de la constante de Michaelis pour la caséine (Shrogart et BECK, I964).

Le poids moléculaire de la protéase de Micrococcus caseolyticus est de $38000 \pm 3$ ooo. Il ne peut être comparé à celui du système protéolytique de $M$. freudenreichii ni à celui de la protéase de Str. faecalis var. liquefaciens. Il est du même ordre de grandeur que celui de la protéase neutre de $B$. subtilis (Tsuru, McConn et Yasunobu, 1965) mentionnée plus haut pour son activité auto-protéo1ytique.

Reçu pour publication en avril 1968.

\section{REMERCIEMENTS}

Nous exprimons notre reconnaissance à M. MOCQUOT pour l'intérêt qu'il a porté à ce travail et pour son aide dans l'établissement du manuscrit.

\section{SUMMARY}

ISOLATION, PURIFICATION AND PROPERTIES OF AN EXTRACELLULAR PROTEINASE FROM " MICROCOCCUS CASEOLYTICUS"

The extracellular proteolytic activity of Micrococcus caseolyticus is due to one proteinase isolated from the culture supernatant. This proteinase was 40 times purified by precipitation with 0,85 -saturated ammonium sulfate, then by negative absorption on DEAE-cellulose ( $0.05 \mathrm{M}$ Tris-maleate buffer), chromatography on DEAE-cellulose ( $0.005 \mathrm{M}$ Tris-maleate buffer), and re-chromatography on Sephadex G-Ioo. The ultracentrifugation pattern of the purified enzyme was homogeneous.

The proteinase has the properties of a metal-enzyme. It is roo per cent inhibited by EDTA, citrates and heavy metal ions. $\mathrm{Ca}^{++}$and $\mathrm{Sr}^{++}$ions induce partial reactivation (60 and $4 \circ$ per cent respectively). Reducing agents induce partial inhibition ; disopropylfluorophosphate and iodoacetic acid induce no inhibition.

Molecular weight estimated on Sephadex G-I00 was $38,000 \pm 3,000$. The $E_{1 \text { an }}^{1 \%}$ at $280 \mathrm{~m} \mu$ was 8.95 at $20^{\circ} \mathrm{C}$ in $0.005 \mathrm{M} \mathrm{Tris}$-maleate buffer at $\mathrm{pH} 7.0$.

The proteolytic activity was much greater. with casein $\left(\mathrm{K}_{m}=0.128 \mathrm{p}\right.$. I00) than with dena- 
turated hemoglobin $\left(\mathrm{K}_{m}=2.2 \mathrm{p}\right.$. Ioo) and $\beta$-lactoglobulin $\left(\mathrm{K}_{m}=9 \mathrm{p}\right.$. 100). The $\mathrm{pH}$ optimum of the enzyme was approximately 7.4. The temperature optimum was $50^{\circ} \mathrm{C}$. The energy of activation was 9,100 $\mathrm{cal} / \mathrm{mole}$.

The proteinase was easily heat-inactivated even with calcium (heat of activation $\Delta H=27 \cdot 36$ $\mathrm{kcal} /$ mole at $50^{\circ} \mathrm{C}$ ). Without calcium the enzyme was stable only in a narrow range of $\mathrm{pH}$ values about 7.0. In $1.5 \times 10^{-3} \mathrm{M}$ calcium chloride, the enzyme was stable from $\mathrm{pH} 6.5$ to $\mathrm{pH} 8.5$.

The lack of stability of the proteinase is due to protein autolysis which is inhibited by calcium.

\section{RÉFÉRENCES BIBLIOGRAPHIQUES}

Akiba T., Fukimbara T., 1967. Studies on the production of acid-protease by submerged culture of Rhizopus. I. Cultural conditions and some properties of the acid-protease. J. Ferment. Technol., 45, 66-7I. ANDREws P., 1964. Estimation of the molecular weights of proteins by Sephadex gel-filtration. Biochem* J., 91, 222-233.

Bleiweis A. S., ZimmermanN L., N., I964. Properties of proteinase from Streptococcus faecalis var. liquefaciens. J. Bacteriol., 88, 653-659.

Colobert L., I957. Étude de la protéinase de Coccus P (Sarcina Aava). I. Purification. Bull. Soc. Chim. Biol., 39, 919-926.

Desmazeaud M., Hermier J., ig68. Facteurs intervenant dans la production du système protéolytique chez Micrococcus caseolyticus. Ann. Biol. Anim. Bioch. Biophys. (sous presse).

Gorini L., I950. Le rôle du calcium dans l'activité et la stabilité de quelques protéinase; bactériennes. Biochim. Biophys. Acta, 6, 237-255.

Hagihara B., Nakai M., Matsubara H., Okuniki K., 1958. I. Preparation of a crystallized protease. J. Biochem., 45, 185-194.

Henri V., 1903. Lois générgles de l'action des diastases. Thèse, Paris.

HuSAin I., MCDONALD I. 1958. Characteristics of an extracellular proteinase from Micrococcus freudenreichit. Can. J. Microbiol., 4, 237-242.

JANSEN E. F., BALLS A. K., I952. The inhibition of $\beta$ - and $\gamma$-chymotrypsin and trypsin by diisopropylfluorophosphate.

Lineweaver H., Burk D., r934. The determination of enzyme dissociation constants. J. Am. Chem. Soc., 58, 658-666.

McCons J. D., Tsuru D., Yasunobu K. T., 1964. Bacillus subtilis neutral proteinase. I. A zinc enzyme of high specific activity. J. Biol. Chem., 239, 3 706-3 715 .

Michaelis L., Menten M. L., I913. Die Kinetic der Invertinwirkung. Biochem. Z., 59, 333-369.

Mizusawa K., Ichishima E., Yoshida F., r964. Studies on the proteolytic enzymes of thermophilic Streptomyces. Part. I. Purification and some properties. Agr. Biol. Chem., 28, 884-895.

MoORE S., STEIN W. H., 1948. Photometric ninhydrine method for use in the chromatography of amino acids. J. Biol. Chem., 176, $367-388$.

Morihara K., 1963. Pseudomonas aeruginosa proteinase. I. Purification and general properties. Biochim. Biophys. Acta, 73, I13-124.

Poznanski S., Lenoir J., Mocquot G., I965. La protéolyse de la caséine par les enzymes intracellulaires de certaines bactéries. Le Lait, 441-442, 3-26.

Shugart L. R., Beck R. W., 1964. Purification and activity of proteinase of Streptococcus faecalis var. liquefaciens. J. Bacteriol., 88, 586-590.

Tsuru D., McConN J.-D., Yasunobu K. T., I965. Bacillus subtilis neutral proteinase. II. Some physicochemical properties. J. Biol. Chem., 240, 2 4r 5-2 420 .

VAllier P., r966. Secrétion d'une protéinase par Micrococcus lysodeikticus (Sarcina flava). Thèse de Doctorat de $3^{\text {e }}$ cycle. Grenoble.

Warburg O., Christian W., r94I. Isolierung und Kristallisation des Gärungsferment Enolase. Biochem. Z., 310, 384-421.

Whitaker J. R., 1963. Determination of molecular weights of proteins by gel filtration on Sephadex. Anal. Chem., 35, 1950-1953.

Wi F. Ch., Laskowski M., 1956. The effect of calcium on chymotrypsins $\alpha$ and $\beta$. Biochim. Biophys. Acta, 19, IIO-I I 5 . 